\title{
Narrativas comunitarias y dinámicas territoriales del proceso de implementación del Acuerdo de Paz en Miranda, Cauca (2016-2018)*
}

DOI: https://doi.org/10.18046/recs.i28.3203

\author{
Community Narratives and Territorial Dynamics \\ of Colombian Peace Agreement Implementation Process \\ in Miranda, Cauca (2016-2018) \\ Narrativas comunitárias e dinâmicas territoriais \\ do processo de implementação do Acordo de Paz \\ em Miranda, Cauca (2O16-2O18) \\ Irene Vélez-Torres ${ }^{* *}$ \\ EIDENAR/Universidad del Valle (Cali, Colombia)
}

\begin{abstract}
* Este esfuerzo de investigación lo dedicamos a David el Mono Marín (Anderson Pérez Osorio), excombatiente de las FARC-EP y líder campesino, quien fue asesinado en Caloto, Cauca, el 17 de junio de 2019. A David lo conocimos en enero de 2017 en Monte Redondo. Estaba seguro de que el camino era la paz y la herramienta, el Acuerdo. Se empeñó en facilitar todo lo que tuviera que ver con educación y comunicaciones en el marco de su implementación. Frente a su asesinato, nos invade una profunda tristeza y una creciente desesperanza. Es así que, con una intención política y académica, este artículo busca que el empeño colectivo de construir la paz no se nos arrebate, al menos no de nuestra memoria.

Este reporte de caso surge del acompañamiento realizado desde finales de 2016 a excombatientes de las FARC-EP y campesinos y campesinas del municipio de Miranda, Cauca, en los primeros años tras la firma del "Acuerdo final para la terminación del conflicto y la construcción de una paz estable y duradera". La investigación fue desarrollada con el apoyo de la Universidad del Valle y el Departamento Administrativo de Ciencia, Tecnología e Innovación (Colciencias). Reporte de caso recibido el 15.08.2018 y aceptado el 29.01.2019.
\end{abstract}

**Correo electrónico: irene.velez@correounivalle.edu.co ORCID: https://orcid.org/oooo-ooo1-8566-6722 


\section{Cómo citar/How to cite}

Vélez-Torres, Irene (2019). Narrativas comunitarias y dinámicas territoriales del proceso de implementación del Acuerdo de Paz en Miranda, Cauca (2016-2018). Revista CS, 28, 153-179. https://doi.org/10.18046/recs.i28.3203 
Resumen

Abstract

Resumo

En cumplimiento del Acuerdo de Paz en Colombia, firmado en 2016, el municipio de Miranda (Cauca) fue uno de los espacios designados para la congregación y dejación de armas de la guerrilla más antigua de América Latina, las FARC-EP. Además, el municipio fue uno de los 170 priorizados por el Gobierno nacional para la implementación de una serie de mecanismos de ordenamiento territorial para la paz, incluyendo el Programa Nacional de Sustitución de Cultivos Ilícitos (PNIS). Este reporte de caso, textual y fotográfico, nace del acompañamiento realizado entre finales de 2016 y 2018 al proceso de implementación de varios de los compromisos firmados entre la guerrilla y el Gobierno. Desde una perspectiva profundamente etnográfica y territorial, este reporte exhibe de manera crítica las narrativas de fracaso que surgieron entre los excombatientes del Espacio Territorial de Capacitación y Reincorporación “Dagoberto Ortiz” en Monte Redondo, así como las dinámicas de las organizaciones sociales e instituciones de Gobierno en el proceso de construcción del Estado durante esta importante transición social y política.

\section{PALABRAS CLAVE:}

guerrilla FARC-EP, campesinos, cultivos ilícitos, dejación de armas, Acuerdo de La Habana

In compliance with the Colombian Peace Agreement, signed in 2016, the municipality of Miranda was one of the designated sites for the congregation and disarming of the oldest guerrilla in Latin America: FARC-EP. In addition, it was one of the 170 prioritized municipalities by the national government for the implementation of a series of mechanisms for territorial planning in the context of peace, including a program that deals with the substitution of the large swaths of coca and marihuana that can be found in the region: the National Program of Substitution of Illicit Crops (PNIS). This case report, textual and photographic, comes from the accompaniment carried out between the end of 2016 and 2018 of the implementation of several of the commitments as signed by the guerrilla and the Colombian government. Based on a deeply ethnographic and territorial 
perspective, this report critically exhibits the narratives of failure that surged within the ex-combats from the Rehabilitation Zone "Dagoberto Ortiz" in Monte Redondo, as well as the dynamics of social organizations and governmental institutions in the process of state-building during this important moment of social and political transition.

\section{KEYWORDS:}

FARC-EP Guerrilla, Peasants, Illicit Crops, Laying Down Weapons, The Havana Peace Agreement

Em conformidade com o Acordo de Paz na Colômbia, assinado em 2016, o município de Miranda, no departamento de Cauca, foi um dos espaços designados para a congregação e o abandono de armas da guerrilha mais antiga da América Latina, as FARC-EP. Além disso, o município foi um dos 170 priorizados pelo governo nacional para a implementação de uma série de mecanismos territoriais de ordenamento da paz, incluindo o Programa Nacional para la Sustitución de Cultivos Ilícitos, (PNIS). Este relato de caso, textual e fotográfico, vem do acompanhamento realizado entre o final de 2016 e 2018 ao processo de implementação de vários dos compromissos firmados entre a guerrilha e o governo. Desde uma perspectiva profundamente etnográfica e territorial, este relatório apresenta criticamente as narrativas de insucesso que surgiram entre os ex-combatentes do Espaço Territorial de Formação e Reintegração “Dagoberto Ortiz” em Monte Redondo, bem como a dinâmica das organizações sociais e instituições governamentais no processo de construção do Estado durante essa importante transição social e política.

\section{PALAVRAS-CHAVE:}

guerrilha FARC-EP, camponeses, cultivos ilícitos, abandono de armas, Acordo de Paz de Havana 


\section{Introducción}

El municipio de Miranda fue uno de los espacios designados para la congregación y dejación de armas de la guerrilla de las FARC-EP. En cumplimiento del punto 3 del Acuerdo de Paz de La Habana (Mesa de Conversaciones, 2017), el cual buscó poner fin al conflicto a través de la dejación de armas y reincorporación de los guerrilleros a la vida civil, desde finales de 2016 se instaló el Punto de Transición y Normalización (PTN) "Dagoberto Ortiz" a donde llegaron más de 220 guerrilleros(as) y milicianos(as) de los frentes Sexto y Galvis a dejar sus armas y a prepararse para su transición a la vida civil. Durante varios meses, los guerrilleros construyeron sus "cambuches" con plásticos negros y verdes -a falta de cumplimiento por parte del Gobierno en el desarrollo de la infraestructura del campamento-, siguieron disciplinadamente sus guardias militares y realizaron reuniones de pedagogía sobre los Acuerdos de Paz. Finalmente, en agosto de 2017 depusieron sus armas.

Demasiado pronto tras la dejación de las armas, los guerrilleros(as) y milicianos(as) se vieron confrontados con la realidad del retraso en el cumplimiento de lo acordado: las promesas de educación, trabajo, acceso a la tierra y establecimiento de proyectos productivos no parecían cumplirse. Pese a las expectativas de los comandantes y de la "guerrillerada", como se le llama a las bases guerrilleras, entre los habitantes temporales del PTN fue creciendo un sentimiento de frustración y traición que se resume en la muchas veces repetida frase: "al Acuerdo le están haciendo conejo". La dejación de las armas, la desintegración de las rutinas militares y la falta de oferta de educación y trabajo transformaron el PTN "Dagoberto Ortiz" en un espacio de ocio que algunos describieron como una cárcel en la montaña.

Transcurrido poco más de un año desde la dejación de las armas, en este Espacio Territorial de Capacitación y Reincorporación (ETCR), nueva denominación del PTN, solo quedan 30 guerrilleros, algunos de los cuales se acompañan hoy de sus esposas e hijos(as). Allí, la única oportunidad productiva que los excombatientes han logrado consolidar es una granja de 5 hectáreas dada en préstamo por una organización campesina: en La Elvira, cerca de 25 excombatientes producen yuca, maíz, fríjol, plátano, tomate chonto y abono bocache, fundamentalmente para el autoconsumo y la comercialización local de los excedentes.

La desintegración de la efímera comunidad guerrillera del ETCR marcó el inicio de un nuevo momento en la implementación del Acuerdo de Paz en Miranda. Funcionarios de distintas agencias y oficinas gubernamentales de los niveles municipal, departamental y nacional comenzaron a desfilar por la alcaldía municipal, por la recién establecida oficina de paz -localizada en una casa vieja al lado de la alcaldía- y por la casa de la cultura. La constitución de distintas mesas de participación 
en torno a los temas de mujer, víctimas, LGBTI, juventud, indígenas y campesinos, sumada a las múltiples reuniones de $\mathrm{PDET}^{1}, \mathrm{PNIS}^{2}$ y CENSO poblacional ${ }^{3}$, así como a las numerosas capacitaciones ofrecidas fundamentalmente por ONG, USAID y la Universidad del Valle, han creado una dinámica de dispersión de la participación social. Pero, sobre todo, ha sido evidente la densificación de la burocracia estatal, en este caso con el pretexto de la implementación del Acuerdo de Paz.

La "reunionitis", como es llamada coloquialmente por los habitantes de Miranda, ha llevado a la mayoría de líderes a criticar la presencia densamente burocrática del Estado y su mínima efectividad en la implementación de lo acordado en La Habana. Una lideresa lo decía con rabia:

¡Esta paz no han sido sino chalecos...! Que venga aquí... Que hable con uno... Que vaya donde el otro... Y al final uno va a ver que no hay es nada: del Acuerdo como tal no hay nada (Lideresa, comunicación personal, O2.2018).

La queja se refiere a la falta de cumplimiento sobre lo comprometido en el Acuerdo de Paz, en particular los puntos uno y cuatro, y sus respectivas disposiciones orientadas a garantizar el acceso a la tierra, los proyectos productivos y la asistencia técnica para la sustitución de cultivos de uso ilícito.

Este reporte textual y fotográfico corresponde al acompañamiento que hemos realizado, de la mano del director audiovisual Sjoerd van Grootheest, al proceso de implementación del Acuerdo de Paz desde finales de 2016 en el municipio de Miranda, Cauca. Por un lado, este acompañamiento se refiere a la intención tanto política como académica de estar presente en el territorio y tejer lazos de solidaridad y confianza con las comunidades locales, con el fin de coparticipar de los procesos sociales surgidos con la implementación del Acuerdo de Paz. Se trata de conocer, desde la cotidianidad de los habitantes civiles y excombatientes, sus visiones, acciones y emociones sobre los cambios que sobrevienen a la dejación de armas de las FARCEP y a los demás procesos articulados a la implementación (o no) de este Acuerdo.

1. Los Programas de Desarrollo con Enfoque Territorial son los instrumentos de planificación y desarrollo territorial para la implementación del Acuerdo de Paz en los municipios priorizados por el Gobierno por su historia de conflicto interno armado (Decreto 893 de 2017 del Ministerio de Agricultura y Desarrollo Territorial).

2. El Programa Nacional de Sustitución de Cultivos Ilícitos es el principal instrumento de gobierno para la sustitución de cultivos de uso ilícito en cumplimiento del punto 4 del Acuerdo de Paz (Decreto 896 de 2017 del Ministerio de Agricultura y Desarrollo Territorial).

3. Corresponde al censo de población y vivienda en realización durante 2018 para contar y caracterizar a todos los residentes, viviendas y hogares en el territorio nacional. 
Por otro lado, el componente visual ha sido central en este acompañamiento y ha respondido al propósito de crear alternativas narrativas que, sin ser escritas, permitan registrar de maneras novedosas las memorias del conflicto armado en esta región. Así, hemos construcción relatos audiovisuales sobre distintos aspectos neurálgicos del proceso de implementación del Acuerdo de Paz, en particular la dejación de armas y la sustitución de cultivos de uso ilícito. En este artículo, sin embargo, las fotografías seleccionadas buscan ilustrar, más desde un estilo periodístico que etnográfico, momentos clave de las dinámicas territoriales reflexionadas en el texto escrito; si se quiere, se trata de usar las fotografías como un correlato ilustrado de los acápites del artículo.

Además, como profesora de la Universidad del Valle un semestre al año (primero de 2017 y primero de 2018), he promovido encuentros entre actores locales del Acuerdo de Paz y estudiantes de pregrado con el ánimo de crear lazos reflexivos y vivenciales entre la comunidad universitaria y los grupos sociales que, en lo local, están llamados a construir paz territorial. Asimismo, he participado de manera constante en distintas reuniones entre las comunidades e instituciones gubernamentales, y he aprovechado mis visitas para adelantar algunas entrevistas y diálogos informales con actores territoriales clave en este proceso de implementación del Acuerdo.

El presente documento puede considerarse un aporte valioso al registro de una memoria no hegemónica sobre la implementación del Acuerdo de Paz y la construcción territorial del estado en el posacuerdo. Es decir, se trata de articular una serie de narrativas y dinámicas territoriales que se distancian del consenso y de la adhesión social que surgió entre el gobierno de Juan Manuel Santos y el Estado Central de las FARC-EP para celebrar la paz. Esta intención dialoga con lo que varios autores han planteado como "otras memorias", que se caracterizan por descentrarse de las víctimas y de versiones válidas y aceptadas sobre el pasado (Calveiro, 2006; Piper-Shafir; Fernández-Droguett; Iñíguez-Rueda, 2013).

Así, la visión que se comparte a continuación disiente de una perspectiva celebratoria y optimista del proceso de implementación del Acuerdo. Aunque se fundamenta en las narrativas de dos grupos sociales que de manera enfática le han apostado a la paz (guerrilleros de las FARC-EP en dejación de armas y campesinos cocaleros en proceso voluntario de sustitución), sus reflexiones muestran una inconformidad y decepción creciente con respecto a los alcances de la paz. Estas perspectivas resultan muy interesantes en la construcción de memorias no hegemónicas del conflicto y la paz, no solo porque hablan desde lo local y desde voces históricamente marginadas y estereotipadas, sino también porque cuestionan profundamente las narrativas de "progreso" en la implementación del Acuerdo (KROC, 2017;2018), mostrando desde una perspectiva cualitativa, que el llamado cumplimiento del mismo se ha centrado 
en la construcción de normas e instituciones, mientras que no se han logrado cambios sustanciales en la vida de la gente.

A continuación, el documento se organiza en cuatro secciones. La primera introduce la aproximación metodológica acogida para adelantar este estudio, el bloque central del texto correspondiente a lo que han sido dos momentos distinguibles en las dinámicas territoriales en Miranda surgidas con la implementación del Acuerdo de Paz durante los primeros veinte meses después de su firma. Así, en la segunda sección del artículo se hará referencia a la situación y las narrativas surgidas en el PTN “Dagoberto Ortiz”, información organizada a través de una crónica personal sobre la situación vivida por los guerrilleros en el transcurso de 2017. Posteriormente, se presentarán algunos testimonios de líderes y lideresas de Miranda, quienes exaltan una visión del Estado como un aparato saturado de funcionarios con escasa voluntad de cumplimiento sobre las obligaciones adquiridas con las comunidades a través de la firma del Acuerdo de Paz. La cuarta y última sección presenta algunas reflexiones sobre los aportes del proceso de investigación.

\section{Aproximación metodológica al caso}

Este reporte textual y fotográfico corresponde al acompañamiento realizado entre finales de 2016 y 2018 al proceso de implementación del "Acuerdo final para la terminación del conflicto y la construcción de una paz estable y duradera” en el municipio de Miranda. Este se ha hecho a través de cuatro estrategias en campo: una colección de entrevistas y diálogos abiertos con exguerrilleros(as) y líderes campesinos; una constante participación en las distintas reuniones entre comunidades e instituciones gubernamentales; el desarrollo y apoyo a procesos formativos con estudiantes y profesores de la Universidad del Valle; y el registro audiovisual de la dejación de armas y del proceso de substitución de cultivos de uso ilícito, bajo la dirección documental de Sjoerd van Grootheest ${ }^{4}$.

Lo que podría llamarse la selección de caso correspondió, realmente, a una combinación entre situaciones intencionadas y fortuitas. Mi trayectoria de investigación -individual y colectiva- se ha centrado en la región del Alto Cauca en Colombia, la cual incluye los municipios más densamente habitados por población afrodescendiente e indígena del norte del departamento del Cauca y sur del Valle del Cauca.

4. El documental "Voces de guerrilla. La desmovilización del Sexto Frente de las FARC-EP" (2018) ha sido ganador del premio al mejor largometraje documental en el Festival Internacional de Cine por los Derechos Humanos y seleccionado en más de diez festivales internacionales. Este narra el proceso de dejación de armas de la guerrilla más antigua de América Latina. Ver trailer en: https://vimeo.com/260395019 
Estos municipios comparten una geohistoria que se teje en torno a la cuenca alta del río Cauca, y que desde 1950 articula conflictos y transformaciones socioecológicas profundas: en la zona plana, estos cambios destructivos se han derivado de la agroindustrialización de la caña; y en la zona de ladera, dichas transformaciones se han asociado a distintas economías ilegales de coca y oro, así como a las disputas por el control territorial armado por parte de grupos de guerrillas, paramilitares y fuerzas armadas del Estado.

En un espacio de formación con mujeres rurales desarrollado en 2016 y centrado en el análisis de conflictos ambientales, una mujer adulta perteneciente a una organización de víctimas del municipio de Miranda me abordó. Me dijo que muy cerca de su vereda habían designado un espacio para congregación de la guerrilla y que ella quería que fuéramos juntas. Previamente habíamos hablado con Sjoerd van Grootheest sobre la relevancia que tendría, en un momento histórico de transición hacia la vida civil, realizar un registro audiovisual de este proceso. Nuestro espíritu era de celebración del recién firmado Acuerdo de Paz, pero también de duda razonable frente a la viabilidad de la implementación, dado el triunfo del "no" en el plebiscito de octubre de $2016^{5}$. Vimos en esta invitación una gran oportunidad para acercarnos al proceso de paz desde el territorio, y tras un par de semanas de reflexión decidimos devolver la llamada a la lideresa. La invitación seguía en pie; y a continuación inició la gestión con los comandantes guerrilleros para programar una primera visita. La historia personal de la lideresa, sobre cómo paramilitares de las AUC y el Frente Sexto de las FARC-EP se enfrentaron por el control territorial en la vereda Guatemala, la supimos en el camino de subida hacia el PTN, un par de semanas después.

Una hora duró la subida en campero y por carretera despavimentada, desde la cabecera municipal hasta Monte Redondo. Atravesamos dos retenes militares fuertemente equipados, en el primero de los cuales fuimos entrevistados a profundidad sobre nuestro destino, proveniencia, afiliación institucional, intención de la visita y nombre de quien nos recibiría en el PTN.

En la vereda, a mano derecha, el primer campamento era de la ONU. Una enorme carpa blanca, muy bien equipada, con visible sistema de aire acondicionado y caminos artificiales alrededor, demarcados con pequeña piedra blanca-con certeza traída

\footnotetext{
5. Por medio de este mecanismo de participación ciudadana, el gobierno del presidente Juan Manuel Santos buscó refrendar el Acuerdo de Paz mediante la pregunta “¿Apoya el acuerdo final para la terminación del conflicto y construcción de una paz estable y duradera?”. Con un total mayor a 13 millones de votos, el "no" se impuso con 50,21\% vs. 49,79\%. A excepción de Bogotá, en todos los departamentos del centro del país ganó el "no", mientras el "sí" se impuso en los departamentos de periferia, donde mayoritariamente se vivió el conflicto interno armado.
} 
desde Cali- que semejaba un conjunto residencial urbano. A menos de 100 metros, un campamento militar se organizaba con sus reconocidas carpas con pintas verdes, también adecuadamente dotado. Pasados otros 100 metros, iniciaba a ambos lados del camino una barrera que, con plástico negro, impedía la visibilidad hacia lo que parecía ser el campamento de los exguerrilleros. Pronto nos explicarían que a un lado se congregaba el Frente Galvis y al otro el Frente Sexto, ambos pertenecientes al Bloque Occidental de las FARC-EP.

Al llegar a lo que intuitivamente reconocimos como el punto de entrada, por ser una casa con un par de personas equipadas con aparatos de comunicaciones en sus manos, parqueamos y nos reportamos. Fuimos informados de que debíamos esperar hasta ser llamados por el comandante. Así lo hicimos. La casa era también una tienda, así que pedimos algunas gaseosas para disimular la ansiedad. Varias personas vinieron en rondas a sentarse a nuestro lado, sin decir palabra, por lapsos de 10 a 15 minutos. Después entenderíamos que eran estrategias de seguridad para escucharnos y observarnos.

Dos horas después, fuimos llamados dentro de la inexpugnable lona negra. Carlos Antonio Acosta, comandante segundo al mando del PTN "Dagoberto Ortiz", nos recibió en compañía de otros dos comandantes de los Frentes Galvis y Sexto. Para sorpresa de todos, el comandante Acosta tenía en su mano la tarjeta de presentación de UniValle que habíamos dejado en el primer retén militar, casi tres horas atrás cuando aún estábamos en la zona plana del valle del río Cauca. Entendimos que su control del territorio era mucho mayor que el imaginado y se daba a través de dispositivos sociomilitares poco obvios. Supusimos también que esas dos horas de espera habían sido usadas para estudiar nuestros perfiles.

Explicamos nuestras intenciones: queríamos filmar la dejación de armas. También queríamos promover un diálogo entre excombatientes y estudiantes de la Universidad del Valle sobre conflictos ambientales relevantes de la región, en particular los temas del agua, la expansión de la frontera agrícola con coca y el modelo agroindustrial. La reunión duró 20 minutos. El comandante Acosta nos preguntó si teníamos financiación. Dijimos que aún no. Nos informó que nos llamarían para confirmar la decisión sobre nuestras propuestas, y así lo hicieron. Un mes después iniciamos las visitas periódicas al PTN.

Sjoerd fue acogido por los excombatientes con increíble apertura. Su condición de hombre extranjero y carisma le permitieron estar en momentos únicos del desarrollo del campamento y de la dejación de armas. Surgió lo que los mismos guerrilleros llamaron una camaradería, que, diríamos, es camaradería para la paz. Conmigo, en cambio, la relación fue siempre más formal o profesional, por decirlo de alguna manera. Yo fui percibida siempre como profesora y experta; y esa condición, aunque daba confianza, mantenía también una distancia en el ámbito personal. 
Con un primer grupo de estudiantes se planteó, durante el primer semestre de 2017, una serie de jornadas de visita al PTN. Allí, los espacios de interacción fueron formales -conversatorios académicos, debates respetuosos y largas jornadas de argumentos sobre temas sensibles como el manejo ambiental y económico de la coca-, pero también lúdicos -los estudiantes organizaron un campeonato de fútbol y una ceremonia de entrega de libros en donación-. Como se aprecia en el video "Pedagogía por la Paz"6, para los estudiantes esta experiencia implicó un completo cambio de visión sobre el conflicto armado en Colombia.

Con un segundo grupo de estudiantes decidimos aproximarnos a los conflictos relacionados con la coca y con el programa de sustitución de cultivos asociado al punto 4 del Acuerdo de Paz. Así, durante el primer semestre de 2018 visitamos varias fincas y dialogamos con campesinos cultivadores de coca y marihuana. La crisis económica generada por el proceso de erradicación sin asistencia técnica fue impactante para los estudiantes, quienes decidieron, como producto de la clase, realizar distintas campañas informativas sobre la situación a través de Facebook y algunos blogs 7 .

Simultáneamente, participamos en algunos de los espacios citados por la alcaldía municipal de Miranda sobre los temas de paz, en los cuales conocimos a otros líderes y lideresas. También, una vez dejadas las armas, acompañamos a algunos de los excombatientes en sus "tareas de pedagogía de paz" en diferentes veredas, lo cual fue una oportunidad para conocer el territorio, sus habitantes y las organizaciones sociales de los municipios de Miranda y Corinto. Durante estos recorridos fuimos invitados por las comunidades a participar de distintas reuniones veredales en que las instituciones gubernamentales abordarían los PDETs, PNIS y el censo poblacional; a estas reuniones llegamos como observadores.

En todo el proceso de campo, la convicción que soporta esta investigación es la de permanecer en el territorio de manera constante y prolongada, vivenciando con las comunidades los desafíos del posacuerdo.

6. El video "Pedagogía por la Paz" (2017) registra el espacio de diálogo creado entre estudiantes de UniValle y comandantes guerrilleros en el proceso de implementación del Acuerdo de Paz. Ver video en: https:// player.vimeo.com/video/221011177

7. Algunas de las piezas comunicativas de los estudiantes se observan en: https://rodriguezdurancarlos.wixsite.com/conflictos y https://www.facebook.com/permalink.php?story_fbid=136737653856405\& $\mathrm{id}=135204870676350$ 


\section{Primer momento: “al Acuerdo le están haciendo conejo”}

Durante el proceso inicial de implementación del Acuerdo, antes de la dejación definitiva de las armas, los excombatientes mantenían en su zona de asentamiento una dinámica de fuerte organización y disciplina militar. Al iniciar nuestras visitas a finales de 2016, y sobre todo entrado el 2017, el primer síntoma del incumplimiento por parte del Gobierno fue el retraso, de al menos seis meses, en el cronograma de construcción de la zona campamentaria. La aparente falta de voluntad se sumó a desaciertos sobre remoción de tierra sin estudios de suelo e incontables improvisaciones en la construcción de los espacios de habitación. Durante más de seis meses los guerrilleros durmieron en cambuches de guadua, plástico y barro, y aunque ya era su costumbre, estas condiciones no cumplían los estándares que les habían prometido; por ejemplo, los baños solo se instalaron en abril de 2017, lo cual implicó problemas de saneamiento y salud pública.

Fото 1 | Armas en el PTN “Dagoberto Ortiz”, junio de 2017

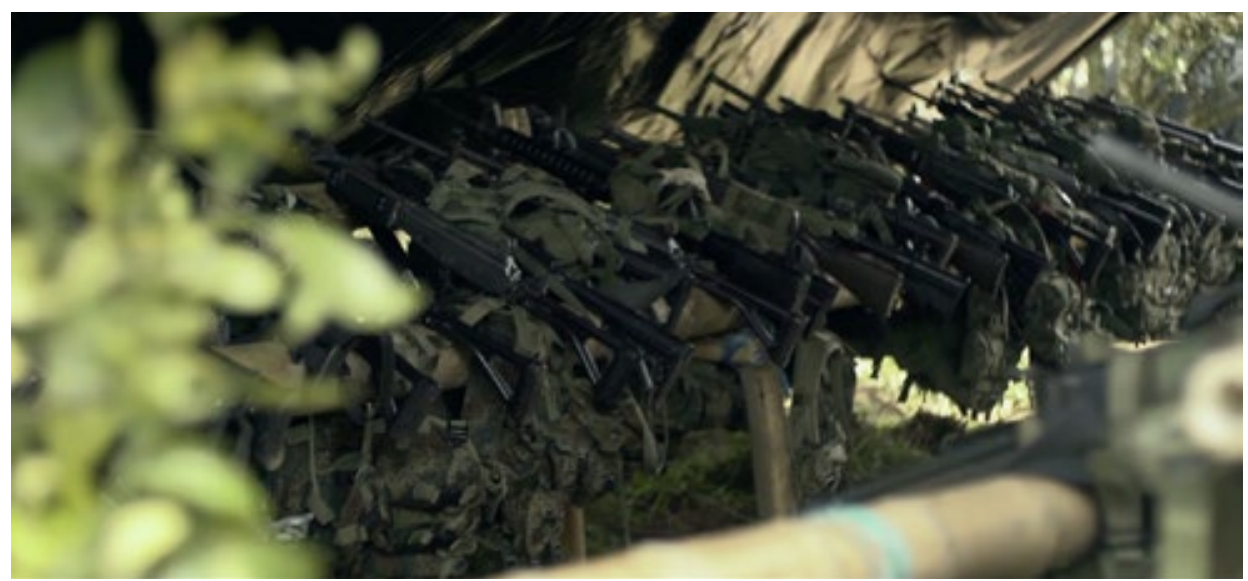

Foto: Sjoerd van Grootheest

Mientras mantuvieron sus armas, los exguerrilleros-aún en camuflaje y en medio de sus guardias matutinas- sentían que tenían un factor de presión sobre el Gobierno para garantizar el cumplimiento de los acuerdos. En todos los espacios colectivos, los comandantes llamaban a la paciencia de la "guerrillerada" sobre la construcción de la zona campamentaria y sobre la tan prometida llegada de las brigadas de salud y de la oferta académica. 
\begin{tabular}{|l|l}
\hline FOто 2 & Parada militar rutinaria en el PTN “Dagoberto Ortiz”, febrero de 2017
\end{tabular}

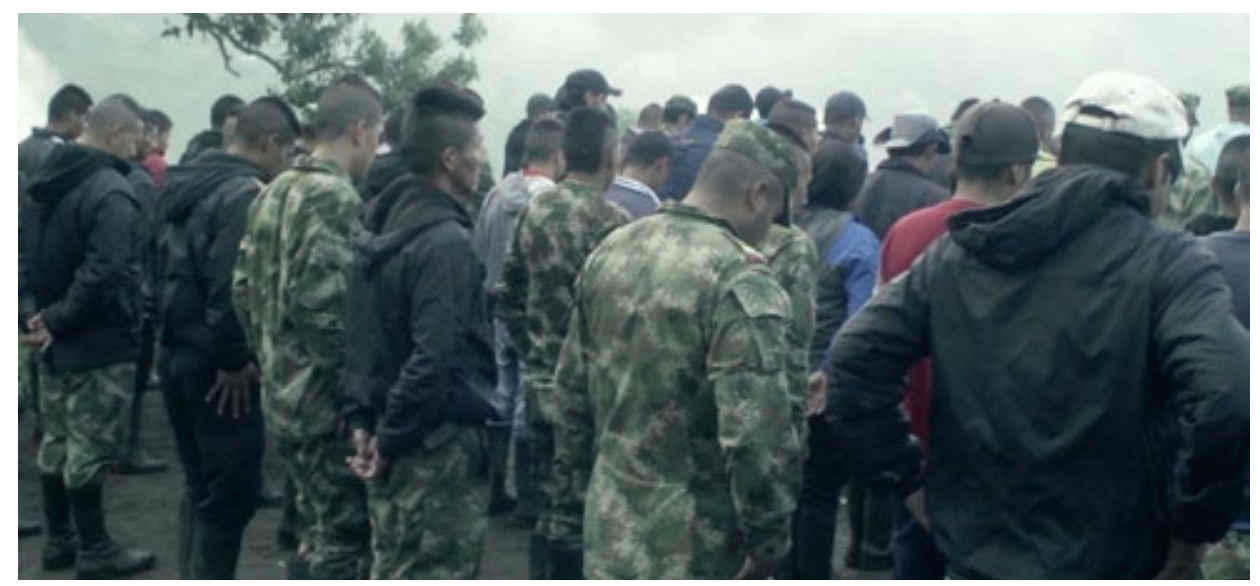

Foto: Sjoerd van Grootheest

Una vez dejaron las armas en agosto de 2017, los exguerrilleros, ahora civiles, no volvieron a recibir materiales de construcción, ni siquiera los faltantes para culminar la construcción de los espacios colectivos en ETCR, así como tampoco han recibido hasta el momento ninguna dotación para los espacios de socialización y formación, como estantería, sillas, mesas, libros o cuadernos. La única construcción que parece completamente terminada en el PTN es la cancha de fútbol que los excombatientes pagaron con sus propios recursos.

Por otro lado, los espacios de habitación fueron finalizados de forma tardía, mediocre y con materiales baratos. Algunas de estas habitaciones presentan a la fecha evidente deterioro, como el quiebre de las láminas de panel-yeso que sirven como paredes, innumerables goteras y desprendimiento de canales de agua lluvia. Los habitantes readecuan estos espacios con sus propios recursos y con los materiales que conocen: guadua y plástico.

La falta de espacios físicos para la socialización y de oferta de servicios para la formación impactó fuertemente la vida cotidiana de los excombatientes y de las pocas familias que desde agosto de 2017 llegaron a vivir ahí. Las personas no encuentran nada que hacer, a excepción de cocinar, ver televisión y jugar uno que otro partido de fútbol los días festivos. Como bien lo dijo un excombatiente: "aquí lo que quieren es matarnos de aburrimiento porque nosotros estamos acostumbrados a mucha actividad y si de repente ya no hay nada que hacer pues uno busca es tirar pa fuera" (septiembre de 2017). Al analizar este testimonio, resulta relevante cuestionar si se 
\begin{tabular}{l|l} 
Fото 3 & $\begin{array}{l}\text { Cancha de fútbol pagada por los guerrilleros del PTN “Dagoberto Ortiz”, } \\
\text { mayo de } 2017\end{array}$
\end{tabular}

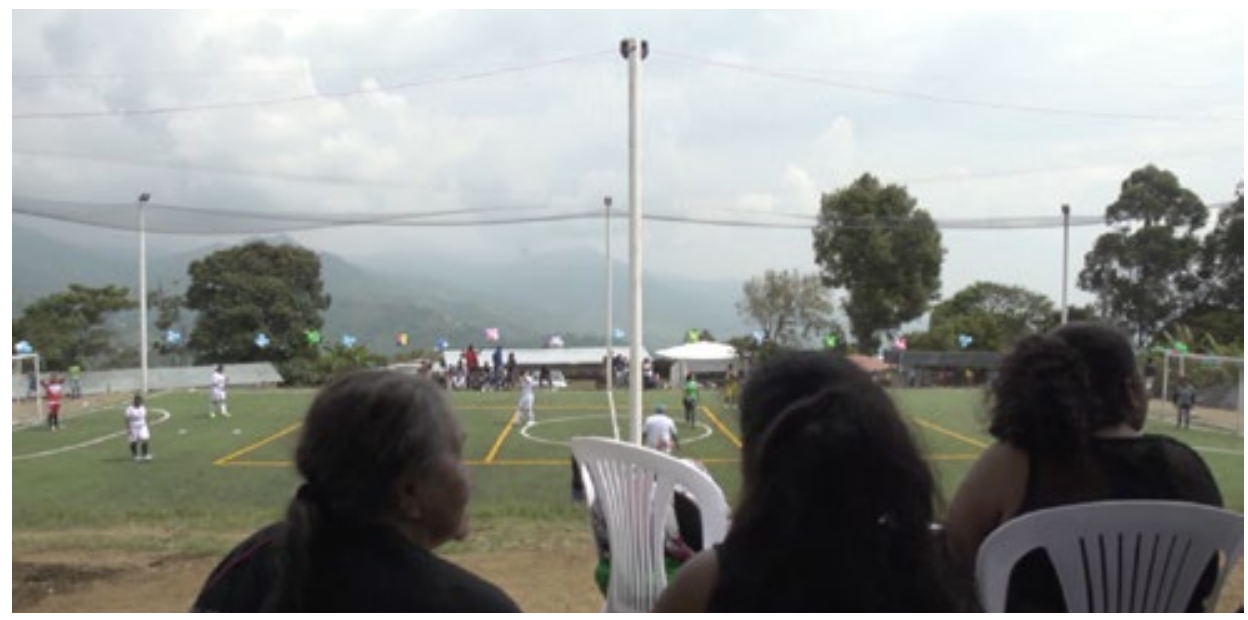

Foto: Sjoerd van Grootheest

Fото 4 Cambuches del PTN “Dagoberto Ortiz”, abril de 2017

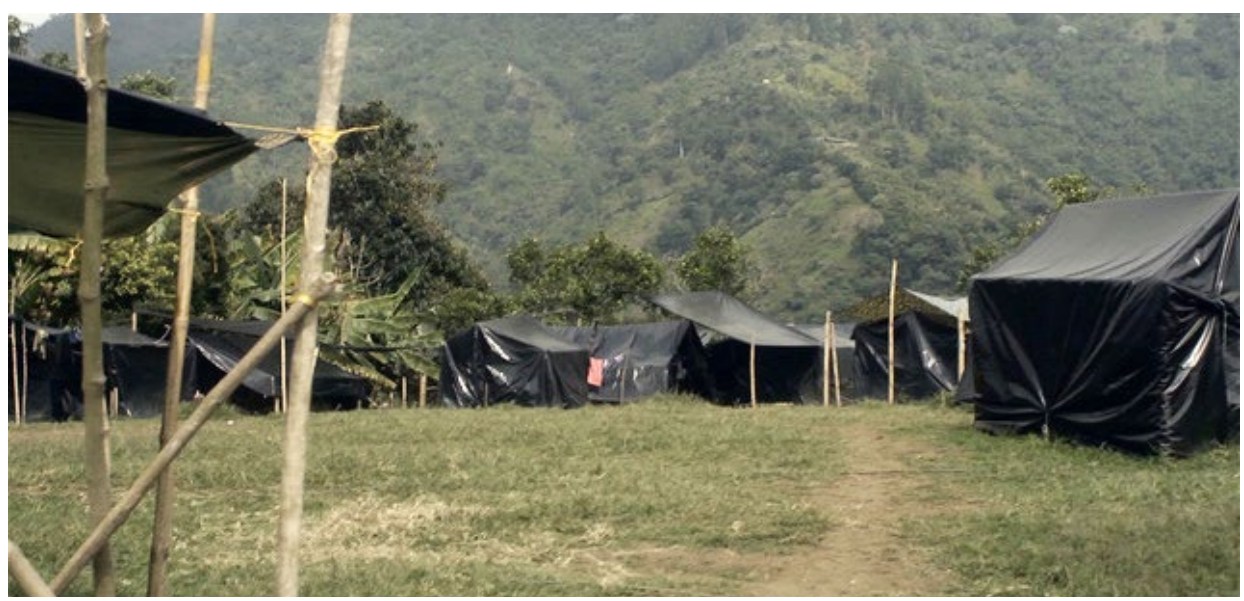

Foto: Sjoerd van Grootheest 
trata acaso del manejo del tiempo como un factor de dominación de la voluntad de quienes llegaron convencidos de aportar a la construcción de la paz.

En estas condiciones, el abandono en desbandada del ETCR no fue más que una consecuencia lógica y necesaria. Como resultado, la efímera comunidad de excombatientes en Monte Redondo se disolvió en menos de un año tras la dejación de las armas. Este proceso fue descrito con resignación y nostalgia por el comandante encargado de los más de doscientos excombatientes de los frentes Sexto y Galvis asentados en esta zona, quien dijo:

Nosotros estamos firmes. Decididos por la paz. Pero yo no puedo obligar a estos muchachos a permanecer aquí sin condiciones. Póngase a ver qué les puedo poner a hacer. Una vez ellos entregaron las armas ya son civiles y pueden hacer lo que esté en sus conciencias. Irán a ver a sus familias al principio y, después, ¿qué habrá para ellos? (...) Los ve uno que este proceso como que no los llenó, como que no les dio lo que esperaban (Comandante, comunicación personal, 03.2017).

Fото 5 | Antiguo PTN “Dagoberto Ortiz”, hoy ETCR, marzo de 2018

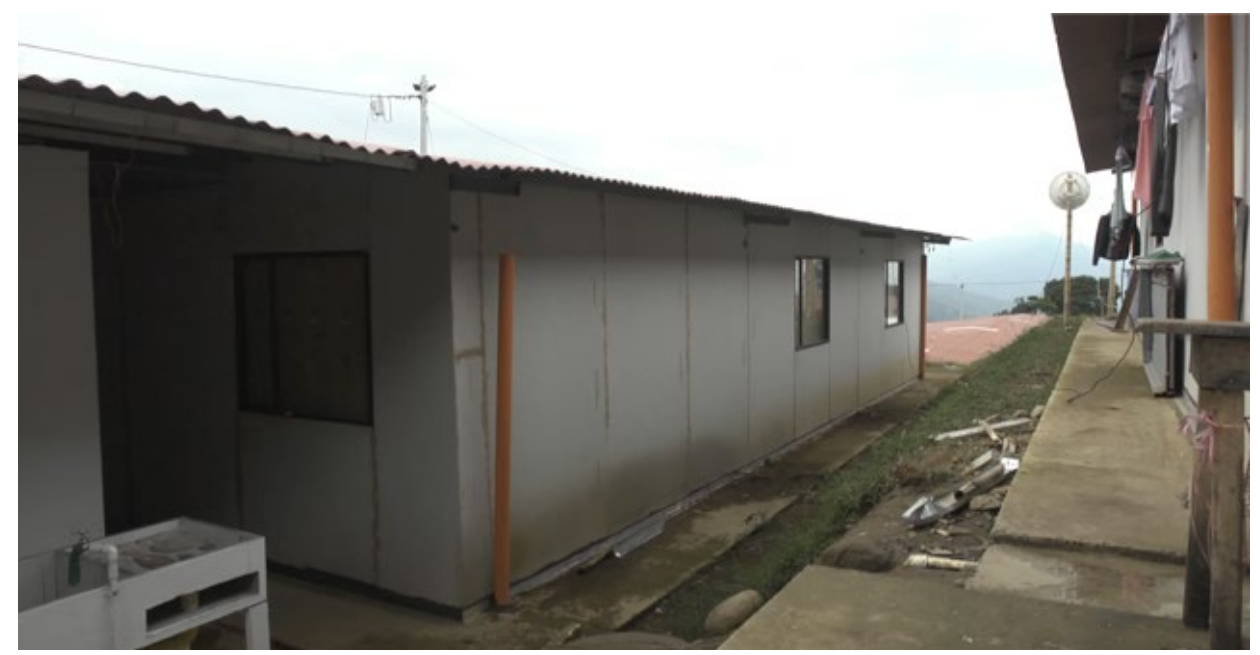

Foto: Sjoerd van Grootheest 
Un testimonio en esta perspectiva lo daba un excombatiente joven que, tras entregadas las armas, les pidió a su esposa e hijo de tres años que vinieran con él al campamento. La mujer accedió a dejar su trabajo como empleada doméstica en Cali y llegó a la zona a principios de septiembre:

Ella me dice que aquí no tienen nada que hacer, que esto no es sino cocine y lave, cocine
y lave. De aquí no podemos salir porque i̇a dónde?! Yo le digo que tenga paciencia, que
tarde que temprano va a llegar algún proyecto y que podemos encontrar qué hacer. Pero
no crea... a mí me da cosa porque a veces me pongo a pensar que esto no tiene ningún
futuro y al final no nos van a salir con nada. Y uno ve: la gente comienza a tomar y eso
tampoco es bueno para los niños (Excombatiente, comunicación personal, 10.2O17).

El ocio fue el resultado de unos proyectos productivos que han sido lentos y de implementación incipiente, así como de una oferta educativa que no llegó. En el momento en que se estableció el campamento, dos profesores voluntariamente se acercaron y se ofrecieron como docentes de música y de carpintería en guadua. Nadie nunca les pagó, así como tampoco hicieron parte de una estrategia del Gobierno.

El Modelo de Nivelación Escolar a excombatientes de las FARC-EP, coordinado por el Consejo Noruego para Refugiados, tuvo una dedicación mínima. Aunque la baja intensidad horaria estaba pensada para que los participantes pudiesen desarrollar otras actividades exigidas por los proyectos productivos, lo cierto es que ante la falta de oferta real de proyectos productivos, esta dedicación escolar resultó insuficiente para ocupar a los excombatientes, a la vez que hizo extremadamente lento el proceso de nivelación.

Pero lo más preocupante frente al proceso formativo es el tipo de educación que recibieron los antiguos guerrilleros. Uno de los comandantes dijo:

A mí me parece una total falta de respeto lo que vienen a darnos. Es que creen que nosotros somos unos brutos, unos idiotas. Nos tratan así, como si fuéramos unos tarados, como si nunca hubiéramos visto la luz (se tapa los ojos con sus dos manos). Eso que nos están dando, cualquiera de aquí (entre los excombatientes) podría darlo ie incluso mejor! Nosotros estábamos esperando algo que nos permitiera subir el nivel. Algo verdaderamente académico... Pero nos salen con esto y usted ¿cómo cree que me siento yo? ¿Cree que no me duele? Si es que nosotros, jugados por la paz, les dijimos a todos estos muchachos que las cosas iban a estar bien para ellos y resulta que ahora ya no tenemos cómo responderles con nada (Comandante, comunicación personal, 10.2017).

La nivelación no cumplió con las expectativas de los excombatientes, además de que ha sido insuficiente para el objetivo de acceder a una educación universitaria, 
pues solo llega hasta grado noveno. En este sentido, no incluye la finalización de la secundaria y presentación de las pruebas SABER, obligatorias para acceder a la educación pública universitaria. Por su parte, otra oferta educativa que llegó tarde fue la de la Universidad del Valle, desde donde hemos ofrecido dos diplomados que solo iniciaron cuando la deserción en la zona había reducido a una tercera parte el número de excombatientes en el ETCR.

Permanecen en Monte Redondo cerca de 30 excombatientes, algunos de los cuales se han acompañado de sus esposas e hijos(as), sumando cerca de 70 habitantes permanentes. La imagen de este espacio es de abandono. No sólo se trata de disidencia. Mayoritariamente la dispersión de lo que fuera un frente guerrillero es producto (i) de una manipulación (intencionada o no) del ocio de los excombatientes y (ii) de una falta de oportunidades concretas de trabajo y acceso a servicios básicos. Dado que el ETCR no logró a tiempo convertirse en un epicentro formativo ni en un espacio productivo, los excombatientes encontraron poco que hacer. A ello se sumó la situación de inseguridad en la zona, lo cual fue llevando a un confinamiento obligado en las instalaciones inacabadas del Espacio Territorial. Como dijera un excombatiente que pasó tres años en un centro penitenciario del Tolima, "lo único que distingue este lugar de la cárcel es la vista. ¡Aparte de eso, aquí ni pasa nada! Ni tampoco uno de aquí puede salir... ¿A quién se le ocurriría salir de aquí como está la cosa?" (Excombatiente, comunicación personal, 02.2018). Quienes a la fecha quedan en el ETCR son solo aquellos que física y socialmente no tienen a dónde más ir.

\section{Segundo momento: "esta paz no han sido sino chalecos"}

Aunque los excombatientes han ratificado su voluntad de acompañar el cumplimiento del punto 4 del Acuerdo de Paz, referido a la sustitución de cultivos de uso ilícito, un comandante dijo de manera enfática que, en las condiciones actuales de incumplimiento, ellos no podían salir a defender una sustitución o una erradicación de cultivos sin garantías, mucho más ahora que la fuerza pública estaba matando a los cocaleros, como fue el caso de los campesinos dedicados a esta actividad y asesinados en Corinto (22 de septiembre de 2017) y en Tumaco ( 7 de octubre de 2017).

Aunque, inicialmente, delegados de las FARC acompañaron los espacios de diálogo entre el Gobierno y los cocaleros como mediadores para lograr acuerdos factibles de sustitución voluntaria, frente al incumplimiento, las FARC retiran su apoyo y los campesinos habrán de afrontar su destino solos. La ausencia de las FARC en los espacios de diálogo y concertación entre campesinos cocaleros y el Gobierno nacional en torno al PNIS fue el primer síntoma de la serie de incumplimientos que experimentarían los campesinos sobre lo pactado en La Habana. 
La economía campesina en el municipio de Miranda, principalmente en la zona de ladera, consiste en una producción limitada de frutales para la comercialización, en especial lulo, mora y granadilla, y una producción extendida de coca y marihuana; también se produce alguna comida para el autoconsumo. La tenencia de la tierra es de pequeñas extensiones, pues la mayoría de familias tiene entre un cuarto y media plaza de tierra, y solo algunas pocas logran la plaza completa; como se sabe, en zona de ladera esta área de tierra es insuficiente para el abastecimiento productivo de una familia, que en promedio tiene cinco integrantes.

Los campesinos y campesinas cuentan que, aunque han venido a ofrecerles algunos palos de granadilla para la siembra, la comercialización aún no está garantizada. En este sentido, un campesino insistía en una entrevista:

Digamos que uno siembra los palitos, pero aquí nadie va a venir a comprar la producción como sí lo hacen con la coca. Con esta carretera, ¿quién se va a asomar por acá? Y pongamos que la bajamos a Miranda (la granadilla), y ahí, ¿quién la va a comprar? No hay. Al mercado uno puede irla a llevar, pero ya sabemos que la compran a pérdida. Los pocos campesinos que ya se han arriesgado a sembrar frutas saben que la cosa es así y le tenemos mucho temor a eso (Campesino, comunicación personal, 10.2017).

La sustitución que se le está proponiendo a la comunidad de campesinos cocaleros no tiene condiciones técnicas ni comerciales que la garanticen. Por ejemplo, los campesinos afirman tener interés en el cultivo de café porque han escuchado de la experiencia exitosa de una finca en la zona, la cual produce una variedad de café especial que se exporta a Bélgica. Pero estos campesinos jamás han cultivado café, no conocen sus requerimientos técnicos ni tampoco han recibido una formación que les permita conocer las formas de cosecha y poscosecha. Como resultado, los campesinos van a la ciega con el futuro productivo de sus fincas.

Antes de firmado el Acuerdo de Paz, los campesinos recibieron recurrentes visitas del Gobierno. Se les prometía que habría inversión en las vías, que los proyectos productivos no tardarían y que tendrían un acompañamiento técnico permanente. $\mathrm{Al}$ respecto, en una de las veredas, una lideresa afirmaba que "después de que las FARC dejó las armas, el Gobierno ya nunca más volvió" (Lideresa campesina, comunicación personal, 11.2017). Y, aunque los campesinos firmaron un preacuerdo voluntario de sustitución de manera colectiva el 20 de agosto de 2017, casi dos años después de la firma de dicho acuerdo de sustitución, los campesinos aún no han recibido la asistencia técnica.

La falta de acompañamiento técnico en la sustitución de cultivos de uso ilícito es particularmente compleja cuando se considera que la mayoría de campesinos en el municipio entraron al programa de sustitución voluntaria y arrancaron sus matas 


$$
\begin{array}{l|l}
\text { FOTо } 6 & \begin{array}{l}
\text { Jornada de firma de acuerdos voluntarios de raspachines recolectores } \\
\text { y cultivadores, vereda La Unión, agosto de } 2017
\end{array}
\end{array}
$$

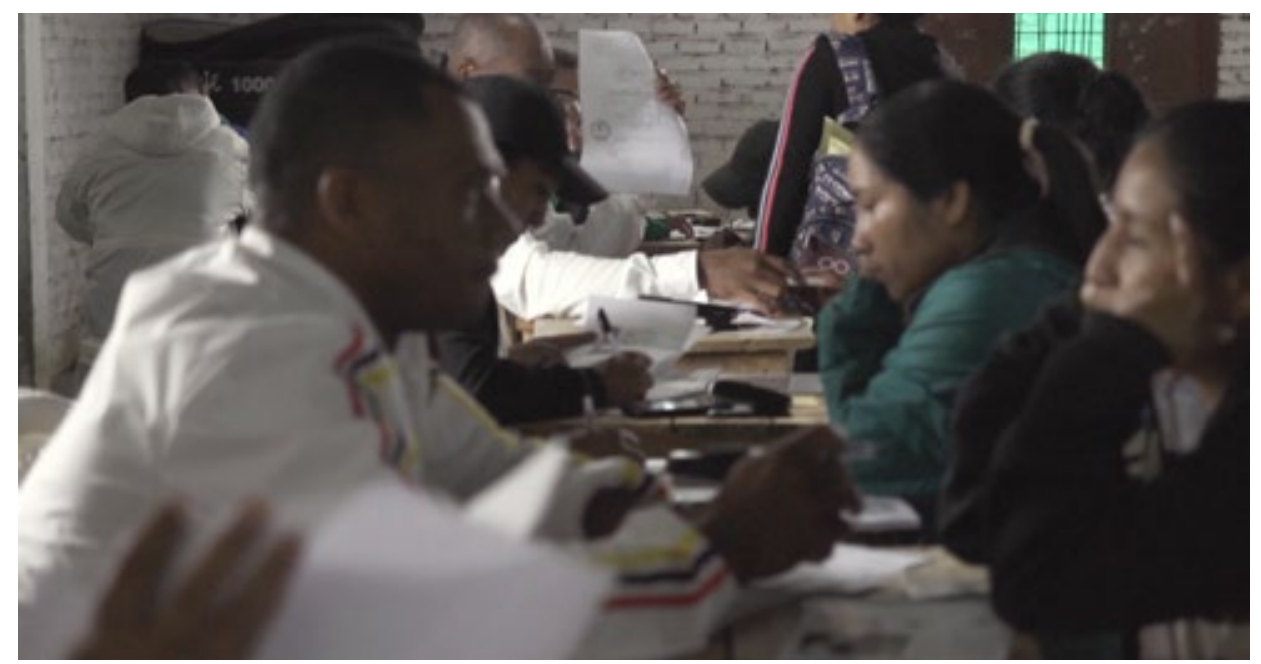

Foto: Sjoerd van Grootheest

de coca con la expectativa de mantener su práctica agrícola campesina, ahora produciendo alimentos y productos legales. Una campesina lo reiteraba en un espacio de diálogo con delegados del PNIS en Miranda: "iEs que comenzaron al revés! Porque esto tenía que venir con el punto 1 de las tierras. Ahora lo que pasa es que nos obligan a sacar la coca y no sabemos nada del futuro que nos espera" (Campesina, comunicación personal, 10.2017).

La gente tiene miedo. Miedo de que lleguen a erradicar de manera forzada a aquellos que, teniendo más de 3 hectáreas de coca, no vieron económicamente viable entrar al programa de sustitución; una erradicación forzada podría generar nuevos enfrentamientos con la fuerza pública y desatar nuevas olas de violencia, como ha sucedido en otras regiones del bajo Cauca y pacífico nariñense desde que se firmó el Acuerdo de Paz. Los campesinos recrean esas realidades vecinas y allí fundamentan sus temores. En una entrevista, abordando el tema de seguridad, un líder de una organización campesina local afirmaba:

Nos han tenido es en reuniones y reuniones de una cosa, reuniones de otra, pero de lo importante nada, solo reuniones tras reuniones. Lo del punto 4 del Acuerdo, reuniones y reuniones, y no se ve nada. Ahora apenas venimos en el punto de caracterización, pero hace ya cuánto que se firmó eso. Hace cuánto que firmaron en Cartagena y mire, apenas estamos, como se dice, comenzando a dar los primeros pasos, pero vemos con 
preocupación de que el Estado habla una cosa y hace otra. Mire allá en Llorente lo que pasó con los compañeros campesinos e indígenas... Allá... Ellos están dentro del punto 4 de la sustitución, porque ellos hicieron el pacto de voluntad de la sustitución y ¿no miró lo que pasó? Les metieron erradicadores, erradicación forzada. Entonces mire, el Estado habla una cosa y hace otra, y esa es la preocupación que nosotros como pueblo tenemos (Líder campesino, comunicación personal, 10.2017).

Los campesinos también reconocen que al haber optado por apoyar el Acuerdo de Paz y haber abanderado territorialmente acciones de pedagogía y de gestión institucional para la implementación, se ha generado una tensión al interior de las mismas comunidades. De manera espontánea, tras un espacio de diálogo entre presidentes de las juntas de acción comunal y funcionarios del Gobierno, un líder de la COCCAM opinaba:

\begin{abstract}
Ahora sí se nos está complicando todo acá. Es que vea, nosotros le dijimos a la gente que era importante defender el Acuerdo y que nosotros sí íbamos a cumplir. Que si el gobierno no cumple, pues que quede claro que nosotros los campesinos sí. Y así nos fuimos haciendo la pedagogía que el Gobierno nunca hizo: hablando con las familias y explicando la ruta (para la sustitución de cultivos de uso ilícito). Pero vea el problema: la gente se atrevió... porque la gente sí quiere ver un cambio. Pero el Gobierno no ha cumplido bien con los pagos, no ha venido a hacer la verificación que falta y ahora ya dicen que la asistencia (técnica) se demora. Entonces, ¿qué dice la gente que ya arrancó sus matas? Pues que es culpa de nosotros porque los metimos en esto (Líder de la COCCAM, comunicación personal, 02.2018).
\end{abstract}

Los campesinos temen que una nueva ola de violencia pueda tener como motor la economía ilegal asociada a la coca y la marihuana, pero también que ello se cruce con nuevos bandos políticos con expresiones armadas. La narrativa de los líderes campesinos revive la historia de haber estado en medio del fuego cruzado, y responsabiliza al Estado de dejarles en medio de dos intereses en contradicción: la sustitución presionada por el Gobierno y la defensa de los cultivos de uso ilícito por parte de algunos productores con áreas de cultivo por encima de las dos hectáreas. Un líder expresaba su preocupación frente al conflicto, a la vez que reivindicaba una aspiración de autonomía territorial desde las guardias Campesina e Indígena:

Esta guerra no se ha acabado. No se ha acabado porque mire, donde los acuerdos estuvieran dando al pie de la letra como deberían de ser, había un dinamismo más para la comunidad, un cambio más rápido para el territorio, pero vemos que no pasa nada. Lo que pasa es como siempre, militarización, militarización. De parte y parte. ¿Sí me 
entiende? De parte de los grupos irregulares como del mismo Estado. Sigue la confrontación y lamentablemente nosotros como comunidad somos los que pagamos el plato roto porque somos los que estamos dentro del territorio y vivimos dentro del territorio, tenemos nuestra familia en el territorio, nuestros hijos. Allí desarrollamos nuestra vida social y somos los que vamos a quedar inmersos nuevamente dentro del conflicto (...). Es que como pueblo nos quieren venir a involucrar dentro del conflicto nuevamente y esto es lo que nosotros no queremos. No queremos nuevamente vernos involucrados en medio de dos fuegos. Por eso le decimos al Estado: no queremos en nuestro territorio ver grupos armados de ninguna índole. Ni estatales ni paraestatales, ni de izquierda ni de derecha. Que nos den a nosotros como comunidad, nos den la oportunidad de nosotros hacernos cargo de nuestro territorio para un mejor desarrollo de las mismas comunidades. Queremos demostrar que no necesitamos de nadie para cuidar lo nuestro y para eso está la guardia (Líder campesino, comunicación personal, 10.2017).

Sobre cuáles pudieran ser las bases políticas de las nuevas violencias en el territorio, otro líder campesino afirmaba:

Porque pues ahora en este momento para nadie es un secreto que, mientras siga la situación así en Colombia, la desigualdad social que hay aquí en el país, mientras que siga el hambre y siga la miseria, pues siempre van a haber personas que se van a querer organizar nuevamente tras las armas. Y es que acá en el territorio ya hay otra gente armada. Acá aparecen grafitis del ELN, el EPL (...). No más el año pasado (2016) en octubre, no llevaban sino quince días allí (los guerrilleros de las FARC-EP) cuando ya aparecen los grafitis de las AUC (Líder campesino, comunicación personal, 10.2017).

El miedo persiste y se alimenta también de la incertidumbre que tienen los y las campesinas frente al cumplimiento institucional. Se trata de una trayectoria de frustraciones en las relaciones institucionales, pero también de la falta de certeza sobre qué sucederá con la economía campesina tras arriesgarse a arrancar las matas de coca y marihuana. Campesinos y campesinas temen que, tras recibir unos pagos, el gobierno "no vuelva a dar la cara" y falte al cumplimiento de los proyectos productivos y de la asistencia técnica, elementos que los campesinos consideran centrales para la continuidad del proceso de sustitución. Cabe anotar que estas son familias pobres a las cuales el cultivo de coca les ha permitido subsistir, pero nunca enriquecerse, es decir que su defensa de este cultivo es la defensa de su principal medio de precaria subsistencia. Nada más y tampoco nada menos.

La burocracia, por otro lado, es una situación dramática que los campesinos refieren como acciones estatales que no aportan en el cumplimiento de lo acordado en La Habana: 
Para nosotros la paz todavía no ha llegado. Prácticamente se firmaron unos acuerdos, donde se tuvo en cuenta la participación del pueblo y pues se mira evidentemente (...), la paz hay que empezarla a construir desde nuestros territorios, hay que empezarla a construir desde la voluntad política de los gobernantes, y ahí es realmente donde no se ha visto (...). Miramos de que a la gente la han cogido ahorita de reuniones tras reuniones, está llegando mucha organización al territorio, una agencia y otra, mucha ONG... Y entonces llaman a los presidentes, a los representantes de las comunidades, que una reunión para el tema de víctimas, que una reunión para el tema del desminado, que una reunión para el tema de los PDET, que una reunión para el tema de la sustitución... Y eso nos pasamos semana tras semana en reuniones, pero realmente no vemos los avances, no vemos los avances de la inversión en los territorios y, fuera de eso, pues vemos de que están matando los campesinos, están matando los indígenas, están matando los compañeros afros (Líder campesino, comunicación personal, 11.2017).

\begin{tabular}{|l|l|}
\hline FOто 7 & $\begin{array}{l}\text { Reunión con tema PNIS en Oficina de Paz de la Alcaldía de Miranda, } \\
\text { abril de } 2018\end{array}$ \\
\hline
\end{tabular}

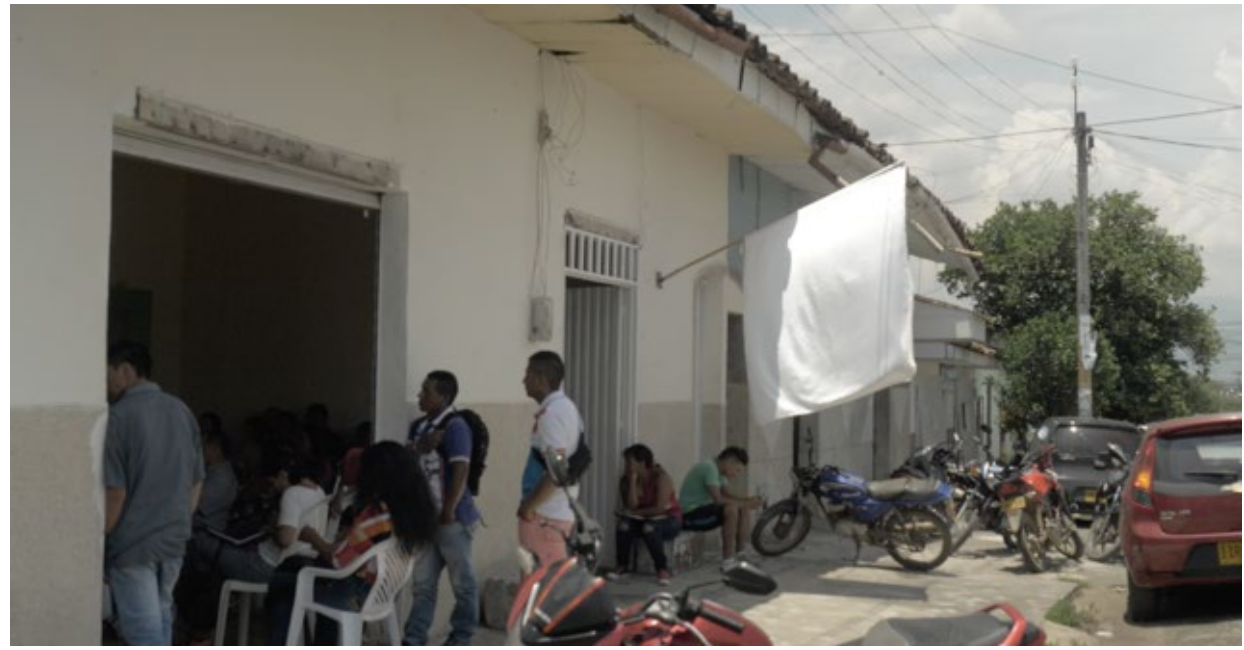

Foto: Sjoerd van Grootheest

La burocracia, envestida de participación social, resuena también con la percepción de inseguridad por parte de los líderes y lideresas. De hecho, una lideresa relacionaba las amenazas recibidas por los principales líderes de las organizaciones sociales de Miranda con su participación en las reuniones convocadas por el Gobierno:

A mí ha comenzado a preocuparme la situación de estas reuniones porque uno va y toma parte de todos esos comités y las reuniones de los programas, pero a veces ni las 
agencias llegan. Uno lo que ve es puro ejército en esas reuniones y bueno, como que se sabe que ahí hay gente que quiere ayudar, pero uno también sabe que esto es zona de guerra y como que han tomado esas reuniones para conocerlo a uno y uno habla y de frente, y allí uno ya no sabe si lo fichan o qué (Lideresa campesina, comunicación personal, 03.2018).

Fото 8 $\quad$ Amenaza a los líderes de Miranda, julio de 2018
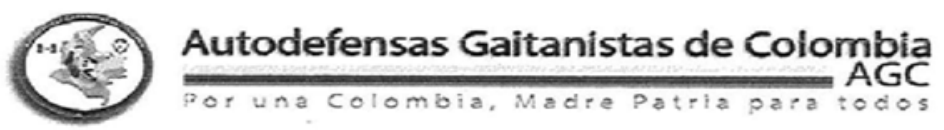

COMUNICADO OPINION PUBLICA

COMUNICADO CLARO Y ESCUENTO DE ALVERTENCIA A DEFENSORES DE DERECHOS HUMANOS LIDERES SOCIALES, AFRODECENDIENTE INDEGENAS Y CAMPESINOS AGRUPADOS EN LA MAL LLAMADAS ORGANIZACIONES CAMPESINAS Y SOCIALES O DE BASE COMO SON: CIMA, PUCSOC, FENSUAGRO, ERPAZ, MESA CAMPESINA. ENTRE OTRAS QUE CREEN EN LA PAZ DEL SEÑOR SANTOS Y QUE VIENEN DESARROLLANDO ACTIVIDADES EN EL MARCO DEL ACUERDO DE PAZ, CON SUS INSERVIBLES AGENCIAS (VICTIMAS) REINCORPORACION SUSTITUCIONAL, RENOVACION TERRITORIAL, PDET Y OTRAS QUE ESTAN EN LOS TERRITORIO DE NUESTRO MANEJO, QUE SON UNA FARZA PARA SEGUIRLE ENTREGANDO EL PAIS A GUERRILLEROS Y AMIGOS DE LA IZQUIERDA CANSERIGENA QUE TANTO DAÑO LE HA HECHO AL PAIS COMO SON LOS FARIANOS Y ELENOS.

QUE APARTIR DE LA FECHA SERAN DECLARADOS OBJETIVO MILITAR QUIENES PARTICIPEN DE ESOS ESCENARIOS DE CHARLAS, REUNIONES, ASAMBLEAS U OTRO TIPO DE CONVOCATORIAS. DE LA MISMA FORMA LE INFORMAMOS QUE FUNCIONERIOS QUE VIENEN CON ESTAS AGENCIAS Y QUE SE HAN UBICADOS EN EL TERRITORIO BIEN SE HAN LOCALES, REGIONALES O NACIONALES TAMBIEN SON NUESTRO OBJETIVO.

CON ESPECIAL ADVERTENCIA A INTEGRANTES DE LA ORGANIZACION DE RESERVAS CAMPESINAS COMO LOS SEÑORES CRISTOBAL JUAMANGA CABEZA PRINCIPAL, EUNER MUÑOS PRESIDENTE, GUSTAVO RENGIFO VICEPREIDENTE Y DEMAS LIDERES, LLAMADOS DEFENSORES DE LOS DERECHOS HUMANOS Y CAMPESINOS CONOCEMOS SU TRABAJO, MOVIMIENTOS, UBICACION Y CADA UNA DE SUS FUNCIONES EN EL MUNICIPIO QUE SIGUEN APOYANDO HA ESCONBATIENTES HPY EL PROCESO DE PAZ.

\author{
JULIO DEL 2018 \\ AUTODEFENSAS GAITANISTAS DE COLOMBIA \\ BLOQUE SUROCCIDENTE \\ PRESENTES
}

\title{
Reflexiones finales
}

El Instituto Kroc fue designado por las partes del Acuerdo de Paz (epígrafe 6.3.2.) para producir una serie de informes que, en tiempo real, dieran cuenta del estado efectivo de la implementación, y algunos patrones emergentes. Su metodología 
se basa en una revisión sistemática del cumplimiento de las 558 disposiciones o compromisos de los que se compone el Acuerdo, los cuales califica en una escala de ninguna actividad encaminada al cumplimiento (o), mínima implementación (1), progreso intermedio (2) o implementación completa (3). Los informes entregados en 2017 y 2018 por el instituto coinciden en dos elementos: afirmar que ha habido un progreso constante en la implementación, valorado en $4 \%$ mensual; y explicar que, comparativamente con otros procesos de paz en el mundo, la tasa de avance de implementación en Colombia corresponde al promedio -ni más, ni menos-.

Aunque ambas afirmaciones pueden ser ciertas, que sean adoptadas por las dos partes firmantes del Acuerdo y convertidas en discurso hegemónico sobre el estado de su implementación, implica desviar la atención respecto a las experiencias vividas en lugares y por poblaciones que, por consiguiente, resultan al margen de la memoria oficial. El consenso creado en Bogotá, en torno a instituciones y mecanismos que antes que democráticos se perfilan burocráticos, conlleva la exclusión de las críticas y narrativas disidentes surgidas desde lo local. Los discursos de las instituciones de Gobierno y de los voceros de FARC, como nuevo partido político, han coincidido hasta la fecha en reforzar el "progreso", aunque lento, de la implementación del Acuerdo. Ello ha descentrado narrativas de actores clave de la guerra y de la transición hacia la paz, sobre todo aquellos que se localizan por fuera del espacio físico y social donde se oficializa la memoria.

En particular, la adhesión social se ha focalizado en la celebración de lo que el Acuerdo ha logrado, por encima de la crítica sobre lo que el mismo ha excluido, lo que en su implementación ha fallado y la dirección hacia la cual se ha orientado su limitado alcance. Esta versión oficial, no obstante, desenfoca el sentir de los excombatientes en dejación de armas, para quienes un panorama de incumplimiento aumenta el riesgo de reinserción armada; también desconoce las experiencias cotidianas de los habitantes rurales más afectados por el conflicto, quienes llenos de esperanza defienden el Acuerdo y, sin embargo, antes que cambios favorables temen el recrudecimiento de una guerra peor que la que les antecede.

Pero la memoria hegemónica y las memorias disidentes son igualmente constitutivas de la historia; ambas son formas de apropiación del pasado (Mate, 2006), inherentes a la construcción del Estado en el contexto del posacuerdo. Mientras la memoria hegemónica se construye desde el centro letrado del poder político, las memorias disidentes gravitan entre visiones, emociones y acciones de sujetos rurales que habitan espacios sociales construidos como periféricos. En consecuencia, identificar y perfilar estas disputas de poder entre las versiones de los actores dominantes y de los marginados resulta crucial para comprender, críticamente, los desafíos que surgen en la transición a la vida civil y en la oportunidad de construir paz territorial. 
Desde una inquietud profunda sobre las disputas políticas que emergen con el Acuerdo y la dirección del cambio que su implementación conlleva, el presente reporte de caso es una herramienta que permite abrir inquietudes y preguntas. En este sentido, se presentan, a continuación, tres reflexiones.

La primera tiene que ver con la posibilidad de que el llamado incumplimiento del Acuerdo se esté haciendo desde su cumplimiento. Aunque suene extraño, este punto se puede explicar a través de un análisis cualitativo sobre las narrativas de cumplimiento. El Segundo Informe del KROC (2018) muestra que el punto 3 contrasta en su porcentaje de cumplimiento con los demás, ya que es el único con un $39 \%$ de cumplimiento completo -los puntos 1, 2, 4 y 5 muestran entre 84 y 95\% de ningún o mínimo cumplimiento-. En este sentido, aunque en la estadística el punto 3 puede parecer ejemplar, el balance presentado por el informe deja ver que las fortalezas están en el ámbito normativo y de creación de instituciones, mientras lo concreto sobre las condiciones efectivas para la reincorporación económica y social (bancarización, acceso a la tierra y recursos para los proyectos productivos) son debilidades reincidentes identificadas entre los informes de 2017 y 2018. Este cumplimiento burocrático del Acuerdo, en lo que se refiere al punto 3, oculta el crítico incumplimiento de las disposiciones capaces de afectar positivamente las causas del conflicto interno armado: el acceso a la tierra, la inequidad, la participación política, entre otras. En este sentido, aunque formalmente es válido afirmar que hay cumplimiento de un número importante de disposiciones, no parecen ser las que más importan a la población beneficiaria de las mismas.

La segunda reflexión tiene que ver con la necesidad de generar un análisis comparativo, no solo sobre la implementación de otros acuerdos de paz en el mundo -como insiste el Instituto KROC-, sino también sobre previos procesos de paz en Colombia (Romero, 2012), así como sobre anteriores intentos legislativos de revertir la inequidad en el acceso a la tierra. Varios autores han planteado que, en Colombia, los procesos de expropiación masiva de tierras se han hecho de forma legal, mientras los intentos de reforma agraria han sido capturados por las élites y consolidados como oportunidades para profundizar el acaparamiento de tierras (Camacho, 2015; Grajales, 2011; LeGrand, 1988; Villar; Ramírez, 2014; Vélez-Torres, 2014). Dada esta configuración del Estado, bajo una alianza histórica entre las élites económicas y los gobiernos, resulta necesario que tanto desde la academia como desde el ejercicio ciudadano se vigile y analice la implementación del Acuerdo y, en especial, del punto 1 que contempla la Reforma Rural Integral. Una mirada crítica sobre nuestra propia historia debe permitirnos anticipar el riesgo de que el Acuerdo de Paz se convierta en una nueva puerta giratoria hacia el despojo y la concentración de la propiedad y del uso de la tierra. 
La última reflexión tiene que ver con la calidad de la participación y, en especial, de la que tiene un enfoque étnico. Lo que en Miranda se ha observado es que, aunque las comunidades afrodescendientes, indígenas y campesinas son invitadas a los espacios de participación de los PDETs y PNIS, los planes vienen prefigurados desde Bogotá y las comunidades tienen una mínima capacidad de maniobra sobre estas definiciones. Por ejemplo, en el caso de las alternativas para sustitución de cultivos de usos ilícito, la oferta local se ha reducido a café, aguacate papelillo y cítricos. Como resultado, el poder de decisión de las comunidades ha terminado reducido al estrecho universo de unos pocos monocultivos tecnificados para la ladera. El mundo imaginado por las comunidades, sus expectativas y sus conocimientos no son ni medianamente explorados por las instituciones, ni mucho menos aprovechados en su enorme riqueza y diversidad socioecológica.

Por un lado, esta situación invita a reflexionar sobre la implementación con perspectiva étnica y la capacidad del Estado para negociar y construir versiones no hegemónicas de desarrollo rural. Por otro lado, este escenario reviste particular importancia en un territorio interétnico donde, desde la década de 1970, las comunidades organizadas han disputado derechos culturales y territoriales diferenciados (Hristov, 2005; Restrepo, 2002). Una comprensión y acción del Estado que no contemple estas visiones e historias de lucha puede agitar los conflictos entre distintos grupos sociales que se enfrentan por acceder a bienes y servicios ambientales que se han hecho escasos en el territorio (Fernández, 2010; Vélez-Torres, 2018). Más aún, una implementación del Acuerdo de Paz que no contemple y, sobre todo, no instituya estos planes propios de ordenamiento del territorio corre el riesgo de llevar a un ejercicio autoritario de un Estado letrado, clasista y centralizado en Bogotá.

\section{Referencias}

Calveiro, Pilar (2006). Los usos políticos de la memoria. En Sujetos sociales y nuevas formas de protesta en la historia reciente de América Latina (pp. 359-382). Buenos Aires: CLACSO.

Camacho, Juana (2015). Paisaje y patrimonio en La Mojana, Caribe colombiano. Geografia Ensino \& Pesquisa, 19, 90-100.

Fernández, Vladimir (2010). Identificación y caracterización de conflictos interétnicos por tierra, territorio y minería en el norte del departamento del cauca. Bogotá: Corporación Andando en Paz, IKV Pax Christi.

Grajales, Jacobo (2011). The rifle and the title: paramilitary violence and land control in Colombia. JPS, 38(4), 771-792. 
Hristov, Jasmin (2005). Indigenous Struggles for Land and Culture in Cauca, Colombia. The Journal of Peasant Studies, 32(1), 88-117.

KROC Institute for International Peace Studies (2017). Estado efectivo de implementación del Acuerdo de Paz en Colombia. Informe 1: Diciembre 2016-Noviembre 2017. Bogotá:University of Notre Dame.

KROC Institute for International Peace Studies (2018). Estado efectivo de implementación del Acuerdo de Paz en Colombia. Informe 2: Diciembre 2016-Mayo 2018. Bogotá: University of Notre Dame.

LeGrand, Cathenne (1988). Colonizacióny protesta campesina en Colombia (1850-1950). Bogotá: Universidad Nacional de Colombia.

Mate, Reyes (2006). Memoria e historia, dos lecturas del pasado. Revista Letras Libres, 53, 44-48.

Mesa de Conversaciones (2017). Acuerdo final para la terminación del conflicto y la construcción de una paz establey duradera. Bogotá: Oficina del Alto Comisionado para la Paz. Recuperado de http://www.altocomisionadoparalapaz.gov.co/herramientas/Documents/AcuerdoFinal-AF-web.pdf

Piper-Shafir, Isabel; Fernández-Droguett, Roberto; Íñiguez-Rueda, Lupicinio (2013). Psicología social de la memoria: espacios y políticas del recuerdo. Psykhe, 22(2), 19-31.

Restrepo, Eduardo (2002). Políticas de la alteridad: Etnización de “comunidad negra” en el Pacífico sur colombiano. The Journal of Latin American and Caribbean Anthropology, 7(2), 34-58.

Romero, Roberto (2012). Unión Patriótica. Expedientes contra el olvido (2. ${ }^{\mathrm{a}}$ ed.). Bogotá: Centro de Memoria, Pazy Reconciliación. Recuperado de http://centromemoria.gov.co/wp-content/ uploads/2015/05/Union-Patriotica-expedientes-contra-el-olvido.pdf

Vélez-Torres, Irene (2014). Governmental Extractivism in Colombia: Legislation, Securitization and the Local Settings of Mining Control. Political Geography, 38, 68-78.

Vélez-Torres, Irene (2018). Una mirada histórica y socio-ambiental para repensar y renombrar los conflictos entre comunidades étnicas del Alto Cauca, Colombia. Ágora USB, 18(1), 38-54.

Villar, Leonardo; Ramírez Juan Mauricio (2014). Infraestructura regional y pobreza rural. FEDESARROLLO - Centro de investigación económica y social. Working paper, 61. Recuperado de:https://repository.fedesarrollo.org.co/bitstream/handle/11445/234/WP_2014_No_61. pdf? sequence $=1$ \&isAllowed $=y$ 